\title{
Milk exosomes: isolation, proteins, and nucleic acids
}

\author{
L. Purvinsh ${ }^{1,2 *}, \mathrm{~S}$. Sedykh $^{1,2}$, G. Nevinsky ${ }^{2}$ \\ ${ }^{1}$ Novosibirsk State University, Novosibirsk, Russia \\ ${ }^{2}$ Institute of Molecular Biology and Fundamental Medicine SB RAS, Novosibirsk, Russia \\ *e-mail:ladapurvinsh13@gmail.com
}

Key words: exosomes, proteins, microRNA

Motivation and Aim: Exosomes are extracellular membrane vesicles with the diameter of $40-100 \mathrm{~nm}$. They are secreted by cells and detected in various biological fluids. Over the past decade, the role of exosomes in many physiological and pathological processes in the body has become clear. The presence of proteins, peptides, DNA, mRNA and, especially, microRNA in their composition is shown, and the role of nucleic acids contained in exosomes in the development of diseases is clarified. The collected data of the exosome composition and functioning serve as the basis for the development of new non-invasive methods for diagnosing various diseases and means of targeted drug delivery in the body. The study of exosomes from milk is very important in the perspective of the development of new approaches to the exosome isolation from complex biological fluids containing a large number of proteins.

Methods and Algorithms: We have developed an approach that combines standard protocol of exosome isolation (ultrafiltration, ultracentrifugation) with additional gel-filtration. According to transmission electron microscopy, this technique allows obtaining homogeneous vesicles. Immunohistochemical staining using antibodies to tetraspanins - the main surface proteins of exosomes was confirmed that the obtained vesicles are exosomes. In addition, the same antibodies were used to create columns for affinity selection of exosomes.

Results: Analysis of protein composition in highly purified milk exosomes showed the presence of a small number of proteins. This indicates that some milk proteins, which were previously described as exosomal, are not part of the exosomes, but co-isolate with them [1]. For the analysis of nucleic acids, we obtained RNA samples at different stages of the exosome isolation, carried out reverse transcription and PCR in real time using primers to several main microRNAs of milk exosomes. Quantitative amplification of this microRNA can serve as an effective high-performance method for determining the content of exosomes in preparations.

Conclusion: The results obtained by us indicate that with the right technology of isolation, milk can serve as a promising source of exosomes necessary for their study and development of new diagnostic approaches and therapeutic drug delivery systems.

\section{References}

1. Sedykh S.E., Purvinish L.V., Monogarov A.S., Burkova E.E. et al. (2017) Purified horse milk exosomes contain an unpredictable small number of major proteins. Biochimie Open. 4:61-72. 